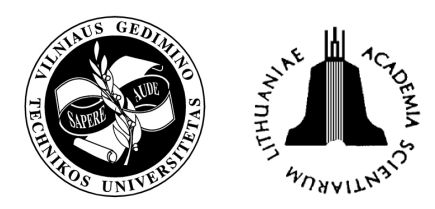

\title{
INVESTIGATION OF POTENTIAL DEFORMATIONS DEVELOPED BY ELEMENTS OF TRANSPORT AND PEDESTRIAN TRAFFIC RESTRICTING GATES DURING MOTOR VEHICLE-GATE INTERACTION
}

\author{
Olegas Prentkovskis ${ }^{1}$, Rasa Prentkovskienè ${ }^{2}$, Ona Lukoševičienė ${ }^{3}$ \\ Vilnius Gediminas Technical University, \\ ${ }^{1,2}$ Dept of Transport Technological Equipment, Plytinès g. 27, LT-10105 Vilnius, Lithuania \\ ${ }^{3}$ Dept of Automobile Transport, J. Basanavičiaus g. 28, LT-03224 Vilnius, Lithuania \\ E-mails: ${ }^{1}$ olegas@ti.vgtu.lt; ${ }^{2}$ rasa@ti.vgtu.lt; ${ }^{3}$ tiauto@ti.vgtu.lt
}

Received 9 January 2007; accepted 2 May 2007

\begin{abstract}
The mathematical model of transport and pedestrian traffic restricting gate is designed. One section of the gate restricted the traffic of motor vehicles while the other limited the traffic of pedestrians. The gate was modelled based on the first-order one-dimensional finite elements taking into account only the resilience of the gate elements and the impact of soil on the ground-embedded parts of the gate support and auxiliary posts. The potential deformations of gate elements were determined based on the mathematical model designed. The specific traffic event was investigated using the mathematical model of gate designed - four situations of motor vehicle-gate interaction were simulated and investigated.
\end{abstract}

Keywords: gate, finite elements, motor vehicle, interaction, deformation, traffic event, computer-run simulation.

\section{Introduction}

The development of motor vehicle transport is obviously a positive point if viewed in terms of social and economic benefits it brings. The penetration rate of motor vehicles is increasing every year and, based on the projections appearing here and there in press, it will only continue to grow. Though viewed within society as a positive trend, this growth of motor vehicle penetration rate, unfortunately, brings about a number of negative factors, too, with the rate of traffic accidents [1-3] standing as the most critical one. Based on the world-wide statistics $[1,2]$, each year, about 700 thousand people perish and another 20 million get injured in traffic accidents. In Lithuania, the Traffic Police record about 6 thousand road accidents per year [4] which appear in the official statistics under any of the following categories:

- vehicle striking a pedestrian;

- vehicle striking a bicyclist;

- collision of running vehicles;

- vehicle rollover;

- collision of vehicle with an obstacle;

- vehicle crashing into another standing vehicle;

- other road accidents.

According to the same statistical data [4], the collision of vehicle with an obstacle accounts for about
$11 \%$ of all traffic accidents recorded in Lithuania. In this case, the obstacle may be represented by a road guardrail, lighting pole, railway switch, tree, gate or any building structure standing close to the road, etc.

In order to restrict the incoming/outgoing traffic of vehicles to/from a particular private or safeguarded territory, there are being used various gates installed in yards as well as on street or automobile roads. The preferences of natural persons and legal entities for one or another type and style of yard gates depend on the trends and demands prompted by the modern style of life. Anyway, whether simple or highly sophisticated, all gates perform the same function - they open or close access to private estates or safeguarded areas. The gates themselves may be made of wood, metal, plastic or a combination of these (Fig 1) [5].

A gate, though viewed as an obstacle subject to deformation, is not attributed to the category of road guardrails even if the structure of some gates could be assumed, based on visual judgment, as corresponding to that of road guardrails. The function of road guardrails is to reduce the probability of motor vehicle violating the traffic by keeping it within the limits of roadway and by guiding its travel along the longitudinal axis of the guardrail and/or fully stopping it $[1,2$, 6]. To enable the aforementioned functions, the guard- 

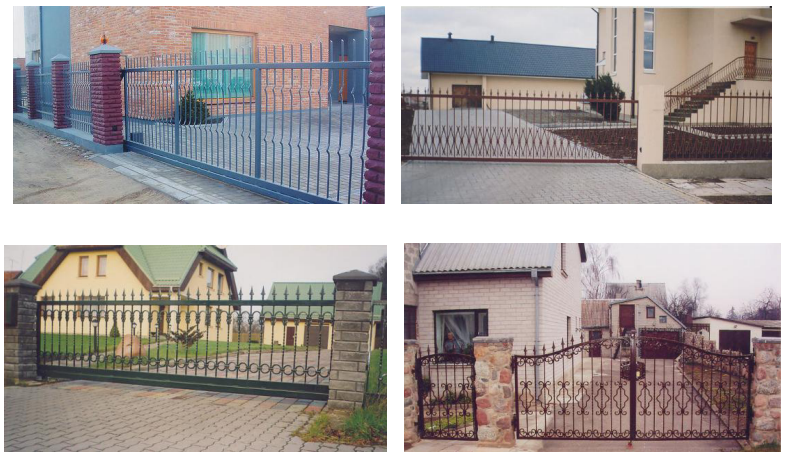

Fig 1. The gates erected to restrict transport and pedestrian traffic

rails are designed in such a way as to ensure full or partial absorption of a motor vehicle kinetic energy thanks to the deformation of guardrail structural elements occurring at certain specific accelerations developed during an impact $[1,2,6]$. Thus, as mentioned before, the function of deforming gates is to restrict transport and pedestrian traffic.

The deformations of gate elements may be investigated using the same simulation models and software as employed for testing the deformation properties exhibited by the elements of road guardrails. The models designed for investigating the deformations of beam guardrail systems may be also successfully applied.

Further the paper presents the case-study of a particular traffic event which took place in one of the Lithuanian cities. As claimed by the participants and witnesses of the traffic event, the motor vehicle did crash into the gate erected at the end of the street to restrict the incoming traffic of motor vehicles and pedestrians to a particular territory.

\section{Overview of research studies}

Worldwide, there exists a great variety of mathematical models and software developed specially for the purpose of investigating deformations of various structural elements. As noted before, the models designed for testing the deformations of road guardrail and beam guardrail system elements appear to be most suitable for testing the deformation properties of gate elements, too.

Let us overview several scientific studies carried out on the subject of deformations:

- The response of a G4(1S) strong post steel Wbeam guardrail system to pendulum impacts has been investigated in a series of full-size physical tests and in simulated experiments using the explicit finite element analysis code DYNA3D. The physical tests were conducted at the Federal Outdoor Impact Laboratory at the Turner Fairbank Highway Research Center of the Federal Highway Administration in McLean, Va. In the pendulum tests, an $880 \mathrm{~kg}$ mass was used to strike the rail perpendicular to its face. The rail section was attached to steel posts and blockouts and supported by a specially designed fixture. Initial velocities of the pendulum at impact were $9.25,20,30$, and $35 \mathrm{~km} / \mathrm{h}$. Acceleration, force, velocity, and displacement histories of the impact event were obtained from accelerometer data taken during the testing. Data from the DYNA3D simulations of the impact tests compared well with the data obtained from the full-scale testing. Displacement plots of the deformed shapes of the rails at $25 \mathrm{~ms}$ intervals compared favorably with high-speed film images. Force versus displacement histories showed good agreement with those obtained from quasi-static experiments. The use of pendulum impact tests for screening and evaluation of alternative guardrail systems is recommended [7].

- A mathematical model of a deforming road guardrail is presented in the paper. Beam metal guardrail is simulated by first order one-dimensional finite elements. Studying the deformation process of the guardrail poles, the impact of soil is taken into consideration. Referring to the mathematical model of a guardrail it is possible to investigate beam metal guardrails mostly used on the automobile roads of Lithuania, to design new guardrails of such type, and also to investigate different transport traffic situations (e.g. the interaction between a motor vehicle and road guardrail). Referring to the model of the guardrail, the bending deflections of deforming elements (metal band, metal poles) of guardrail are investigated [6].

- The authors of this paper focus on the simulation of the motor vehicle movement (taking into consideration motor vehicle dynamics, motor vehicle hydraulic brake system influence on motor vehicle movement, interaction between its wheels and road pavements, road guardrail characteristics, interaction between motor vehicle and road guardrail) on a certain road section and propose their specific solution to this problem. The presented results illustrate the motor vehicle movement trajectories (motor vehicle braking and interaction between motor vehicle and road guardrail at various initial conditions and at various certain pavement surface of the road section under investigation) and work of a motor vehicle hydraulic brake system. Taking into consideration the presented general mathematical model and computer aided test results it is possible to investigate various road transport traffic situations as well as to investigate various transport traffic safety problems [8].

- Guardrail posts made of recycled plastics are becoming candidates for use in roadside safety features due to the nature of exhibiting properties that are useful in absorbing energy. Relatively few studies have specifically addressed the performance of recycled guardrail posts in strong-post guardrail systems. Few have reported success in this area. In this study, a detailed computer simulation study is performed to evaluate the crashworthiness of the 
recycled content guardrail posts. Results of a fullscale crash test are also used to verify the accuracy of the finite element models used in the simulation study. Based on the investigation, it is demonstrated that computer simulations can effectively model the dynamic response behaviour of a crash event and provide valuable information about the impact performance of recycled content guardrail posts. Crash simulation results indicate that when proper quality recycled posts are used the guardrail system has the potential for meeting the National Cooperative Highway Research Program Report 350 recommendations [9].

- A procedure for reconstructing run-off-road accidents into longitudinal W-beam guardrail systems was developed by estimating energy dissipation during an impact. Correlations were developed between the vehicle's departure angle, velocity, type of vehicle, and the energy dissipated. Energy losses are due to barrier-vehicle friction; post/soil deformations; guardrail beam deformations, and vehicle energy losses attributed to the vehicle (this is composed of actual vehicle damage and tireground friction, with vehicle damage being predominated). Barrier-vehicle friction losses were found to range from about 5 to $36 \%$, depending upon speed and impact angle. The energy to cause permanent deflection of the guardrail posts in a system was found to be roughly equivalent to the amount of energy dissipated by the rail deformation of that system. Comparisons with full-scale crash test results showed that the developed iterative reconstruction procedure accurately estimated impact velocities within $3 \%$ [10].

- Strong-post round-wood W-beam guardrail, $\mathrm{G} 4(\mathrm{RW})$, is a common longitudinal barrier used in Texas. Recent crash tests on the system demonstrate its effectiveness in containing and redirecting a $2000 \mathrm{~kg}$ pickup truck. Even though the guardrail performed satisfactorily according to National Cooperative Highway Research Program Report 350 requirements, there were concerns about the stability of the vehicle due to excessive wheel snagging on the posts. In this study, a modification to the existing design is evaluated to improve the stability of an impacting vehicle. LS-DYNA, a dynamic, nonlinear, large deformation finite element modelling code is used to analyze and compare the performance of alternate designs. A baseline finite element model of the existing system is developed to replicate the observed crash test behaviour of the G4(RW). The fidelity of the baseline model is verified against full-scale crash test results. To improve the vehicle stability, the current design was modified to include guardrail posts with shorter embedment depth. An optimum post embedment depth was determined and used in the second simulation study. Finite element analysis results show that an improved G4(RW) system eliminates wheel snag- ging on posts, reduces sudden vehicle deceleration and therefore improves the stability of an impacting vehicle significantly [11].

- Pavement mow strips are being used to combat growth of vegetation around guardrail posts. However, the effect of pavement post encasement on crashworthiness of strong post guardrail systems has not been investigated. In this paper, performance of these systems is examined using experimental testing and numerical simulation. Mow strip dimensions, materials, and depths are considered in addition to the presence of "leave-out" sections around posts. Seventeen configurations using wood and steel posts embedded in various mow strip configurations and confinement conditions were subjected to dynamic impact testing with a bogie vehicle. Dynamic impact tests were numerically simulated and full-scale mow strip system models were assembled using the subcomponent models. A concrete mow strip with grout leaveouts was designed based on predictive numerical simulations. This design was subsequently constructed and subjected to full-scale crash testing. With reference to nationally accepted criteria, crash tests of a strong post steel guardrail system and a wood post guardrail system encased in the selected mow strip configuration were considered to be successful. Recommendations for implementation are provided [12].

- This article presents results from numerical simulations of bumper beam systems, important structures of an automobile that provide passenger protection from front and rear collisions. This investigation explores bumper beam - longitudinal systems that are subjected to $40 \%$ offset impact loading. The numerical simulations use the nonlinear finite element code LS-DYNA to observe system behaviour. In addition, a comparative study of an industrial-like modelling procedure is performed that includes user-defined material such as state-of-the-art aniosotropic plasticity, an isotropic strain, a strain rate hardening rule, and ductile fracture criteria. Numerical crash results agree with the experiments, regarding overall deformation mode and energy dissipation. The simulations give relatively accurate predictions of the collapse mode found in the experimental tests. An exception occurs with one bumper beam - longitudinal system that has AA7003-TI longitudinals. The research also includes sensitivity studies that consider both physical (e.g., strain-rate effects, heat-affected zone) and numerical (e.g., adaptive meshing) parameters [13].

- For longitudinal barriers, it is common practice to use standard W-beam guardrail along the required highway segments and to use a stiffened Thrie-beam guardrail in a transition region near the end of a bridge. As a result of the differences in rail geometries, a W-beam to Thrie-beam transition element is 
typically used to connect and provide continuity between the two rail sections. However, the W-beam to Thrie-beam transition element has not been evaluated according to current impact safety standards. Therefore, an approach to guardrail transition system, including a W-beam to Thrie-beam transition element, was constructed and crash tested. The transition system was attached to Missouri's Thriebeam and channel-bridge railing system [14].

The studies covered in the above overview, however, represent only a very small share of all the extensive research efforts existing world-wide and devoted to herein addressed subject. Some studies are conducted using very costly hardware and software whereas others require the performance of ordered onscene experiments which, too, are very expensive.

The authors of this publication tried to carry out the investigation of the selected traffic event and the analysis of potential deformations on the elements of transport and pedestrian traffic restricting gates developed during motor vehicle-gate interaction as least-cost and accurate as possible. The models applied for investigating this particular case are presented in the $[2,6,8]$ papers.

\section{Brief record of traffic event}

As will be mentioned in Introduction, a particular traffic event took place in one of the Lithuanian cities.

While examining the traffic event, the authors of this publication identified the presence of some contradictory facts.

On the street road concerned, there was installed a gate restricting the traffic of motor vehicles and pedestrians. The scheme of the gate is presented in Fig 2. The gate was of the type which could rotate around the pivot of the gate support post and rest against the auxiliary post while in closed position. The gate support and auxiliary posts were erected on the street pavements (Fig 3). One section of the gate (the longer one) restricted the traffic of motor vehicles while the other (the shorter one) limited the traffic of pedestrians.

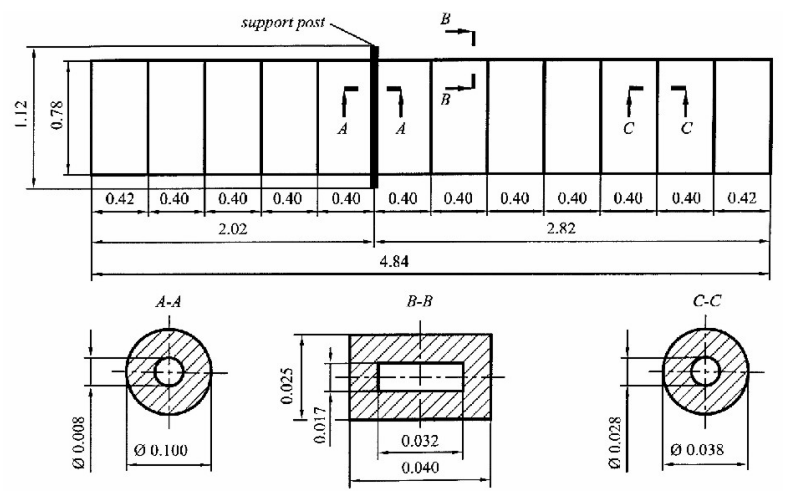

Fig 2. The scheme of the gate concerned

Neither the gate nor the posts were found in place after the traffic event. Later, the gates were found and they appeared to contain some queer deformations. The a)

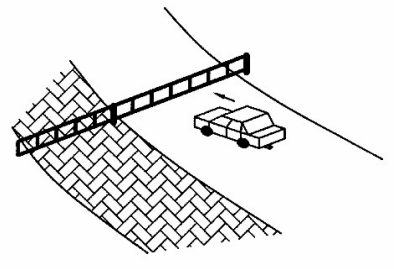

b)

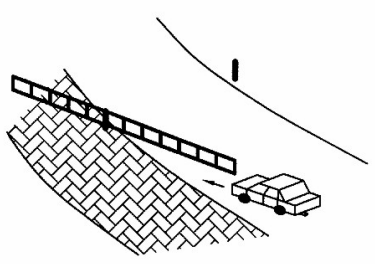

c)

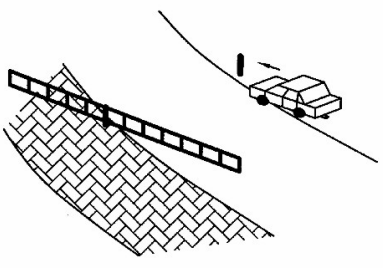

Fig 3. The scheme of motor vehicle-gate interactions

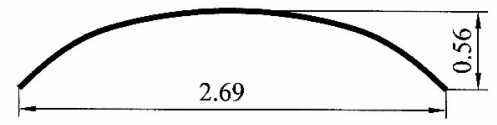

Fig 4. Deformations identified on the section of gate which restricts the traffic of motor vehicles (the top-view)

section restricting the traffic of motor vehicles was found with its arch symmetrically deformed (Fig 4). The ground-embedded remains of the posts stayed in place but the posts themselves were missing producing the effect as if they had been accurately truncated (they were not found on the scene). The section of the gate which restricted the traffic of pedestrians was not found, either.

\section{Mathematical model of gate}

The potential deformations of gate elements were investigated using the Finite Element Method. The mathematical gate model was developed based on the mathematical model of road guardrail $[2,6,8]$.

The gates were modelled using the first-order onedimensional finite elements (Fig 5) [2, 6]. At the moment of an impact, the nodes of finite elements change their position within the system of $X-Y-Z$ coordinates (a gate develops deformations). Therefore, while modelling, only the resilience of gate elements as well as soil impact upon the ground-embedded parts of the gate support and auxiliary posts were taken into consideration $[2,6]$.

Since a gate is a mechanical system, the system of finite element movement equations was drawn based on the Langrangian second-order equations [2, 6, 8]. 
a)
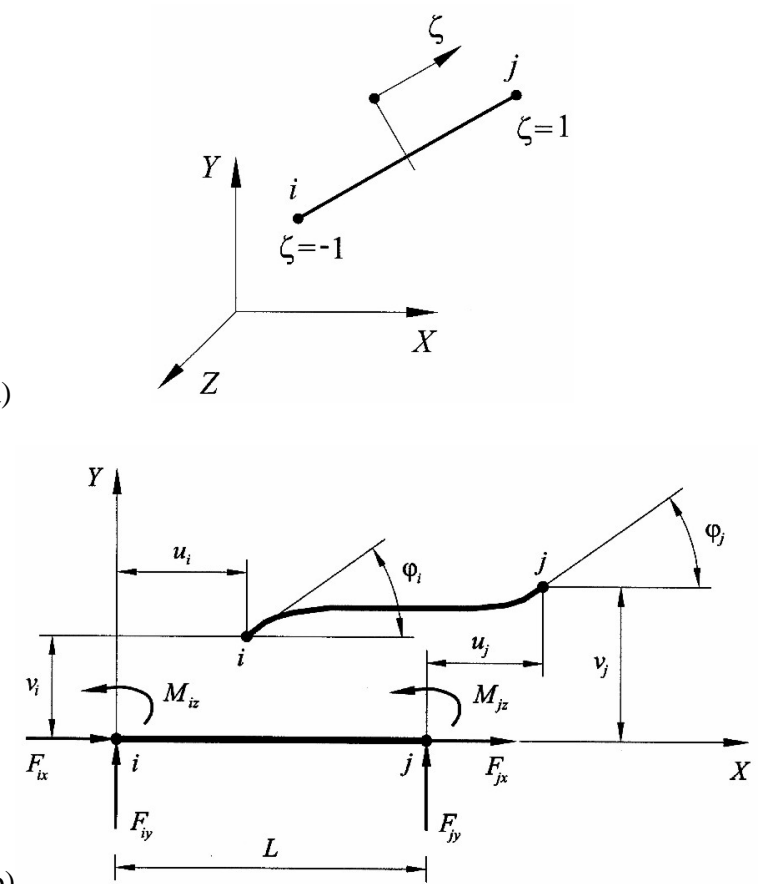

Fig 5. The first-order one-dimensional finite element: a - the scheme within the system of $X-Y-Z$ coordinates; $\mathrm{b}$ - the deformations on $X-Y$ plane

Considering the fact that a displacement on a finite element is approximated:

$$
\left\{u^{(e)}\right\}=[N]\left\{q^{(e)}\right\}
$$

where: $\left\{u^{(e)}\right\}$ - finite element displacement; $[N]$ - finite element shape functions; $\left\{q^{(e)}\right\}$ - vector of finite element generalized displacements.

The following expressions were entered for the finite element:

- the kinetic energy;

- the potential energy;

- the dissipative function;

- the vector of external forces of impact on the gate finite element.

By entering the above-listed expressions into the Langrangian second-order equation, we obtained the following matrix pattern for the system of finite element movement equations:

$$
\left[M^{(e)}\right]\left\{\ddot{q}^{(e)}\right\}+\left[C^{(e)}\right]\left\{\dot{q}^{(e)}\right\}+\left[K^{(e)}\right]\left\{q^{(e)}\right\}=\left\{F^{(e)}\right\},
$$

where: $\left\lfloor M^{(e)}\right\rfloor,\left\lfloor C^{(e)}\right\rfloor,\left\lfloor K^{(e)}\right\rfloor$ - matrixes of finite element masses, mechanical energy damping and stiffness; $\left\{\ddot{q}^{(e)}\right\},\left\{\dot{q}^{(e)}\right\},\left\{q^{(e)}\right\}-$ vectors of finite element generalized accelerations, velocities and displacements; $\left\{F^{(e)}\right\}$ - vector of finite element generalized forces.

Further, all systems of finite element movement equations were combined together to form the following general system of gate movement equations:

$$
\begin{aligned}
& {\left[M_{\text {gate }}\right]\left\{\ddot{q}_{\text {gate }}\right\}+\left[C_{\text {gate }}\right]\left\{\dot{q}_{\text {gate }}\right\}+} \\
& {\left[K_{\text {gate }}\right]\left\{q_{\text {gate }}\right\}=\left\{F_{\text {gate }}\right\},}
\end{aligned}
$$

where: $\left[M_{\text {gate }}\right],\left[C_{\text {gate }}\right],\left[K_{\text {gate }}\right]-$ matrixes of gate masses, mechanical energy damping and stiffness; $\left\{\ddot{q}_{\text {gate }}\right\},\left\{\dot{q}_{\text {gate }}\right\},\left\{q_{\text {gate }}\right\}$ - vectors of accelerations, velocities and displacements for all gate nodes; $\left\{F_{\text {gate }}\right\}-$ vector of generalized forces of impact on the gate:

$$
\begin{aligned}
& {\left[M_{\text {gate }}\right]=\sum_{e=1}^{N E}\left[M^{(e)}\right] ;\left[C_{\text {gate }}\right]=\sum_{e=1}^{N E}\left[C^{(e)}\right] ;} \\
& {\left[K_{\text {gate }}\right]=\sum_{e=1}^{N E}\left[K^{(e)}\right] ;\left\{F_{\text {gate }}\right\}=\sum_{e=1}^{N E}\left\{F^{(e)}\right\} ;} \\
& \left\{\ddot{q}_{\text {gate }}\right\}=\sum_{e=1}^{N E}\left\{\ddot{q}^{(e)}\right\} ;\left\{\dot{q}_{\text {gate }}\right\}=\sum_{e=1}^{N E}\left\{\dot{q}^{(e)}\right\} ; \\
& \left\{q_{\text {gate }}\right\}=\sum_{e=1}^{N E}\left\{q^{(e)}\right\},
\end{aligned}
$$

where: $N E$ - number of finite elements.

The matrixes of finite element masses, mechanical energy damping and stiffness were set considering the performance of finite element deformation on the planes $X-Y$ (Fig $5 \mathrm{~b}$ ) and $X-Z$ as well as the relation between the local and global systems of coordinates [2].

\section{Outcomes of computer-run simulation}

The computer-run simulation was performed on a personal computer using the Maple [15] and Compaq Visual Fortran software [16]. The matrixes of finite element masses, mechanical energy damping and stiffness were generated in the Maple software environment whereas the mathematical model of gate was developed using the Compaq Visual Fortran software.

As noted before, the mathematical model was designed specially for the purpose of investigating the particular traffic event.

We determined the force of impact upon the gate $F_{\text {gate }}$ based on the available speed value of the motor vehicle concerned and then added this force at certain nodes of the gate finite elements.

The following four situations of motor vehiclegate interaction were simulated and investigated:

- The motor vehicle and the horizontal beam of the gate interact on the horizontal plane along the transverse axis of the gate (Fig 3 a, the gate is tightly closed). The computational scheme and the outcomes of computer-run simulation are presented in Fig 6 below.

- The motor vehicle and the vertical beam of the gate (fixed between the two horizontal beams) interact on the vertical plane along the transverse axis of the gate (Fig 3 a, the gate is tightly closed). The computational sche- 


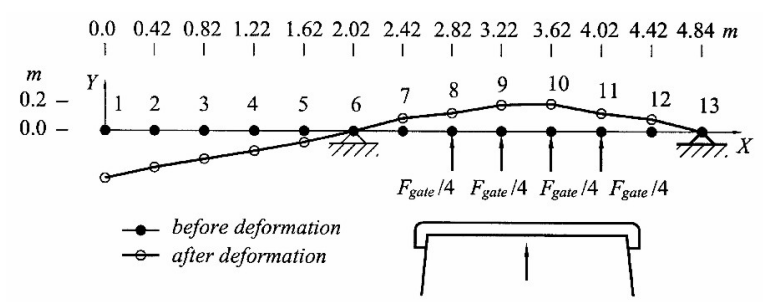

Fig 6. The computational scheme and the outcomes of computer-run simulation

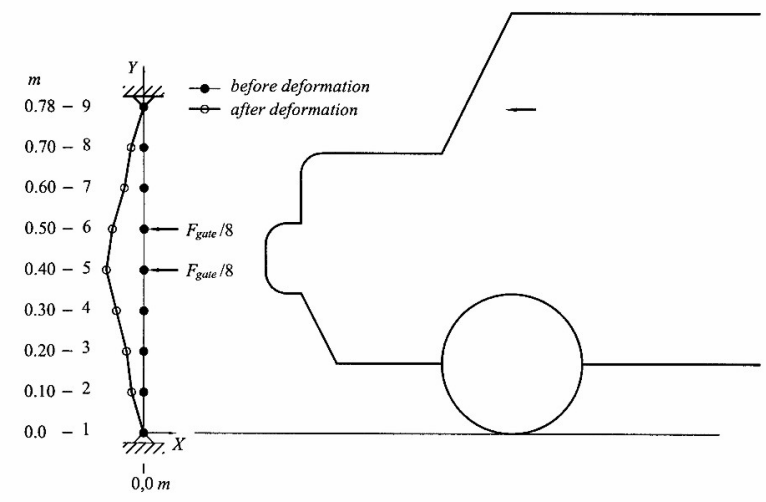

Fig 7. The computational scheme and the outcomes of the computer-run simulation

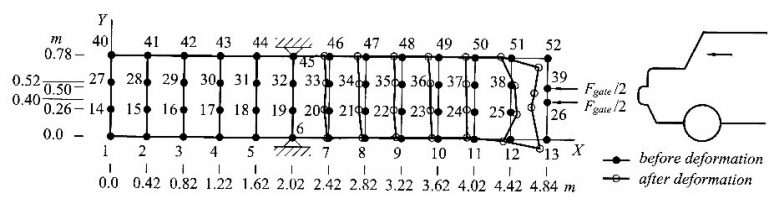

Fig 8. The computational scheme and the outcomes of the computer-run simulation

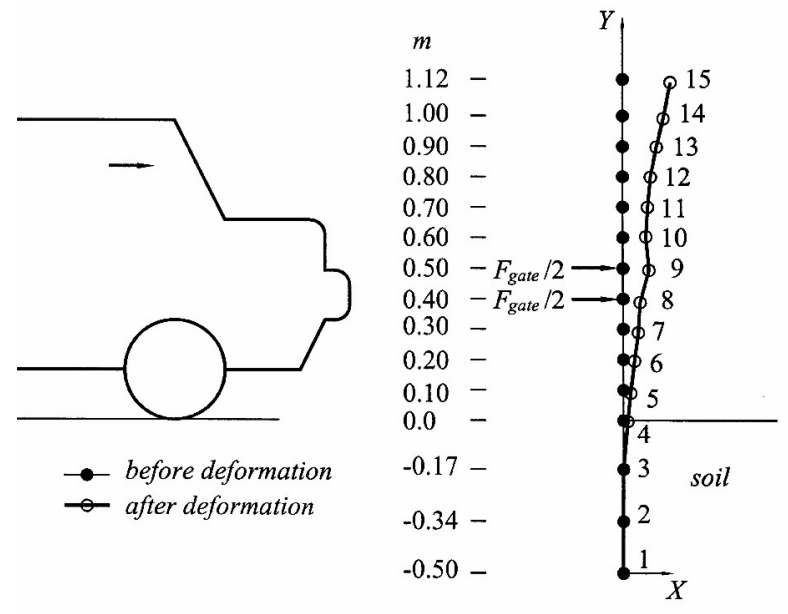

Fig 9. The computational scheme and the outcomes of the computer-run simulation me and the outcomes of the computer-run simulation are presented in Fig 7 below.

- The motor vehicle and the gate interact on the vertical plane along the longitudinal axis of the gate (Fig $3 \mathrm{~b}$, the gate is wide open). The computational scheme and the outcomes of the computer-run simulation are presented in Fig 8 below.

- The motor vehicle and the gate auxiliary post interact on the vertical plane (Fig $3 \mathrm{c}$, the gate is wide open). The computational scheme and the outcomes of computer-run simulation are presented in Fig 9 below.

\section{Conclusions}

1. The mathematical model of transport and pedestrian traffic restricting gate is designed.

2. The gate was modelled based on the first-order onedimensional finite elements taking into account only the resilience of the gate elements and the impact of soil on the ground-embedded parts of the gate support and auxiliary posts.

3 . The potential deformations of gate elements were determined based on the mathematical model designed.

4. The specific traffic event was investigated using the mathematical model of gate designed. Based on the investigation results, it was concluded that the impact of motor vehicle on the gate had not actually occurred.

5. The mathematical model of gate presented may be applied (alone or in combination with other models) for investigating and simulating other traffic events, too.

6. The computer-run simulation (for the purpose of working out the mathematical model of gate) was performed using specially developed Maple and Compaq Visual Fortran Professional software-based applied programs.

\section{References}

1. BAUBLYS, A.; BOGDEVIČIUS, M.; PRENTKOVSKIS, O. et al. Transport: technologies, economics, environment, health: monograph (Transportas: technologijos, ekonomika, aplinka, sveikata: monografija). Vilnius: Technika, 2003. 876 p. (in Lithuanian).

2. PRENTKOVSKIS, O. Interaction between the vehicle and obstacles: summary of doctoral dissertation (technological sciences, transport engineering). Vilnius: Technika, 2000. $56 \mathrm{p}$.

3. PRENTKOVSKIS, O. The anniversary: the Journal "TRANSPORT" - 20 years together! Transport, 2006, Vol 21, No 4, p. IIa-IIc.

4. www.lra.lt - website of the Lithuanian Road Administration under the Ministry of Transport and Communications of the Republic of Lithuania.

5. www.eurolaikas.lt - website of Company "Eurolaikas".

6. BOGDEVIČIUS, M.; PRENTKOVSKIS, O. Simulation of road guardrails. Transportas (Transport Engineering). Vilnius: Technika, 2001, Vol 16, No 4, p. 123-128. 
7. BANK, L. C.; YIN, J.; GENTRY, T. R. Pendulum impact tests on steel W-beam guardrails. Journal of Transportation Engineering, July/August 1998, Vol 124, No 4, p. 319-325.

8. BOGDEVIČIUS, M.; PRENTKOVSKIS, O.; VLADIMIROV, O. Engineering solutions of traffic safety problems of road transport. Transport, 2004, Vol 19, No 1, p. 43-50.

9. ATAHAN, A. O.; ROSS, H. E. Computer simulation of recycled content guardrail post impacts. Journal of Transportation Engineering, November/December 2004, Vol 130, No 6, p. 733-741.

10. COON, B. A.; REID, J. D. Crash reconstruction technique for longitudinal barriers. Journal of Transportation Engineering, January 2005, Vol 131, No 1, p. 54-62.

11. ATAHAN, A. O.; CANSIZ, Ö. F. Improvements to G4(RW) strong-post round-wood, W-beam guardrail system. Journal of Transportation Engineering, January 2005, Vol 131, No 1, p. 63-73.

12. SECKINGER, N. R.; ABU-ODEH, A.; BLIGH, R. P.; ROSCHKE, P. N. Performance of guardrail systems encased in pavement mow strips. Journal of Transportation Engineering, November 2005, Vol 131, No 11, p. 851-860.

13. KOKKULA, S.; HOPPERSTAD, O. S.; LADEMO, O.-G.; BERSTAD, T.; LANGSETH, M. Offset impact behaviour of bumper beam-longitudinal systems: numerical simulations. International Journal of Crashworthiness, 2006, Vol 11, No 4, p. 317-336.

14. POLIVKA, K. A.; COON, B. A.; SICKING, D. L. et al. Midwest guardrail system W-beam to Thrie-beam transition. In: TRB 86th Annual Meeting Compendium of Papers CD-ROM (Transportation Research Board Annual Meeting 2007. Paper No. 07-2628). 2007. 20 p.

15. ALADJEV, V.; BOGDEVIČIUS, M.; PRENTKOVSKIS, O. New software for mathematical package Maple of releases 6, 7 and 8: monograph. Vilnius: Technika, 2002. $404 \mathrm{p}$.

16. SCHICK, W.; SILVERMAN, G. Fortran 90 and engineering computation. John Wiley \& Sons, 1995. 425 p. 